Proyecciones

Vol. 27, No 1, pp. 103-111, May 2008.

Universidad Católica del Norte

Antofagasta - Chile

\title{
ON CONMUTATIVE LEFT-NILALGEBRAS OF INDEX 4 *
}

\author{
JUAN C. GUTIERREZ FERNANDEZ \\ UNIVERSIDADE DE SAO PAULO, BRASIL
}

Received : Diciembre 2007. Accepted : March 2008

\begin{abstract}
We first present a solution to a conjecture of (Correa, Hentzel, Labra, 2002) in the positive. We show that if $A$ is a commutative nonassociative algebra over a field of characteristic $\neq 2,3$, satisfying the identity $x(x(x x))=0$, then $L_{a^{t_{1}}} L_{a^{t_{2}}} \cdots L_{a^{t_{s}}} \equiv 0$ if $t_{1}+t_{2}+\cdots+t_{s} \geq 10$, where $a \in A$.
\end{abstract}

Keywords : solvable; commutative; nilalgebra;

Mathematics Subject Classification : 17A05, 17A30

\footnotetext{
${ }^{*}$ Partially Supported by FAPESP 05/01790-9, Brasil
} 


\section{Introduction}

Throughout this paper the term algebra is understood to be a commutative not necessarily associative algebra. We will use the notations and terminology of (Fernandez, 2004). Let $A$ be an (commutative nonassociative) algebra over a field $F$. We define inductively the following powers, $A^{1}=A$ and $A^{s}=\sum_{i+j=s} A^{i} A^{j}$ for all positive integers $s \geq 2$. We shall say that $A$ is nilpotent if there is a positive integer $s$ such that $A^{s}=(0)$. The least such number is called the index of nilpotency of the algebra $A$. The algebra $A$ is called nilalgebra if given $a \in A$ we have that $\operatorname{alg}(a)$, the subalgebra of $A$ generated by $a$, is nilpotent. The (principal) powers of an element $a$ in $A$ are defined recursively by $a^{1}=a$ and $a^{i+1}=a a^{i}$ for all integers $i \geq 1$. The algebra $A$ is called left-nilalgebra if for every $a$ in $A$ there exists an integer $k=k(a)$ such that $a^{k}=0$. The smallest positive integer $k$ which this property is the index. Obviously, every nilalgebra is left-nilalgebra. For any element $a$ in $A$, the linear mapping $L_{a}$ of $A$ defined by $x \rightarrow a x$ is called multiplication operator of $A$. An Engel algebra is an algebra in which every multiplication operator is nilpotent in the sense that for every $a \in A$ there exists a positive integer $j$ such that $L_{a}^{j}=0$.

An important question is that of the existence of simple nilalgebras in the class of finite-dimensional algebras. In (Fernandez, 2004) we proved that every nilagebra $A$ of dimension $\leq 6$ over a field of characteristic $\neq 2,3,5$ is solvable and hence $A^{2} A$. For power-associative nilalgebras of dimension $\leq 8$ over a field of characteristic $\neq 2,3,5$, we have shown in (Fernandez, Suazo, 2005) that they are solvable, and hence there is no simple algebra in this subclass. See also (Elgueta, Suazo, 2004; Fernandez, 2004) for power-associative nilalgebras of dimension $\leq 7$.

We show now the process of linearization of identities, which is an important tool in the theory of varieties of algebras. See (Gerstenhaber, 1960; Osborn, 1972; Zhevlakov, 1982) for more information. Let $P$ be the free commutative nonassociative polynomial ring in two generators $x$ and $y$ over a field $F$. For every $\alpha_{1}, \ldots, \alpha_{r} \in P$, the operator linearization $\delta\left[\alpha_{1}, \ldots, \alpha_{r}\right]$ can be defined as follows: if $p(x, y)$ is a monomial in $P$, then $\delta\left[\alpha_{1}, \ldots, \alpha_{r}\right] p(x, y)$ is obtained by making all the possible replacements of $r$ of the $k$ identical arguments $x$ by $\alpha_{1}, \ldots, \alpha_{r}$ and summing the resulting terms if $x$-degree of $p(x, y)$ is $\geq r$, and is equal to zero in other cases. Some examples of this operator are

$$
\begin{array}{rc}
{[l l] \delta[y]\left(x^{2}(x y)\right)} & =2(x y)^{2}+x^{2} y^{2} \\
\delta\left[x^{2}, y\right]\left(x^{2}\right) & =2 x^{2} y, \quad \delta\left[y, x y^{2}, x\right]\left(x^{2}\right)=0 .
\end{array}
$$

For simplicity, $\delta[\alpha: r]$ will denote $\delta\left[\alpha_{1}, \ldots, \alpha_{r}\right]$, where $\alpha_{1}=\cdots=\alpha_{r}=\alpha$. We observe that if $p(x)$ is a polynomial in $P$, then $p(x+y)=p(x)+\sum_{j=1}^{\infty} \delta[y: j] p(x)$, where $\delta[y: j] p(x)$ is the sum of all the terms of $p(x+y)$ which have degree $j$ with respect to $y$.

Lemma 1. (Zhevlakov, 1982) Let $p(x, y)$ be a commutative nonassociative polynomial 
of $x$-degree $\leq n$. If $F$ is a field of characteristic either zero or $\geq n$, and the $F$-algebra $A$ satisfies the identity $p(x, y)$, then $A$ satisfies all linearizations of $p(x, y)$.

\section{Left-nilalgebras of index 4}

Throughout this section $F$ is a field of characteristic different from 2 or 3 and all the algebras are over $F$. We will study left-nilalgebras of index $\leq 4$, that is the variety $V$ of algebras over the field $F$ satisfying the identity

$$
x^{4}=0 .
$$

Let $A$ be an algebra in $V$. For simplicity, we will denote by $L$ and $U$ the multiplication operators, $L_{x}$ and $L_{x^{2}}$ respectively, where $x$ is an element in $A$. The following known result is a basic tool in our investigation. See (Correa, Hentzel, Labra, 2002; Elduque, Labra, 2007).

Lemma 2. Let $A$ be a commutative left-nilalgebra of index 4 . Then $A$ satisfies the identities

$$
x x=-x(x x), \quad x x=(x)^{3}=x(x(x x)),
$$

and $p(x)=0$, for every monomial $p(x)$ with $x$-degree $\geq 7$. Furthermore, we have

$$
\begin{aligned}
{[l c l] L_{x} } & =-L U-2 L^{3} \\
L_{x x^{2}} & =-U-2 U L^{2}-2 L U L+4 L^{4}, \\
L_{x(x x)} & =-L U-2 L U L-2 L U L-4 L^{3} U-12 L^{5}, \\
L_{x(x(x x))} & =2 L U+4 L U L+4 L^{4} U+8 L^{6},
\end{aligned}
$$

and also

Table i. Multiplication identities of degree 5. \begin{tabular}{ccccccccc}
\cline { 2 - 7 } & & $U L U$ & $L U^{2}$ & $U L^{3}$ & $L U L^{2}$ & $L^{2} U L$ & $L^{3} U$ & $L^{5}$ \\
\hline$U^{2} L$ & 0 & -1 & 2 & 0 & 0 & -2 & -8 \\
\hline
\end{tabular}

and two identities of $x$-degree 6 which may be written as

Table ii. Multiplication identities of degree 6.

\begin{tabular}{lccccccccc}
\hline & $U L^{2} U$ & $(L U)^{2}$ & $L^{2} U^{2}$ & $U L^{4}$ & $L U L^{3}$ & $L^{2} U L^{2}$ & $L^{3} U L$ & $L^{4} U$ & $L^{6}$ \\
\hline$U^{3}$ & -2 & -2 & 2 & -8 & -8 & 0 & -4 & 8 & 40 \\
$(U L)^{2}$ & -1 & -1 & 1 & -4 & -2 & 2 & 0 & 4 & 24 \\
\hline
\end{tabular}

We note that, for example, Table i means that $U^{2} L=-L U^{2}+2 U L^{3}-2 L^{3} U-8 L^{5}$. From the identities (2.3-2.6) we get that for any $a \in A$ the associative algebra $A_{a}$ generated by all $L_{c}$ with $c \in \operatorname{alg}(a)$ is in fact generated by $L_{a}$ and $L_{a^{2}}$. Furthermore, every algebra in $V$ is a nilalgebra of index $\leq 7$. 
We now pass to study homogeneous identities in $A$ with $x$-degree $\geq 7$ and $y$-degree 1. From the relation $0=\delta[y, x, x, x]\left(x^{4}\right)=2 y\left(x\left(x^{3} x^{3}\right)\right)+4 y\left(x^{3}\left(x x^{3}\right)\right)+2 x\left(y\left(x^{3} x^{3}\right)\right)+$ $4 x^{3}\left(y\left(x x^{3}\right)\right)+4 x\left(x^{3}\left(y x^{3}\right)\right)+4 x^{3}\left(x\left(y x^{3}\right)\right)+4 x^{3}\left(x^{3}(x y)\right)=2 x\left(\left(x^{3}\right)^{2} y\right)+4 x\left(x^{3}\left(x^{3} y\right)\right)+$ $4 x^{3}\left(x\left(x^{3} y\right)\right)+4 x^{3}\left(x^{3}(x y)\right)=2\left[L L_{x^{3} x^{3}}+2 L L_{x^{3}} L_{x^{3}}+2 L_{x^{3}} L L_{x^{3}}+2 L_{x^{3}} L_{x^{3}} L\right](y)$ we have

$$
L^{3} U^{2}=-2 L^{3} U L-L^{4} U L-5 L^{5} U-20 L^{7}
$$

since we can use the reductions (2.3-2.6) and replace the occurrences of $(U L)^{2}$. Multiplying the identity of Table i by $U$ from the left, replacing first the occurrences of $U^{3}$ and next using reductions from Table i, Table ii and above identity we get a new identity as follows:

$$
\begin{aligned}
0= & U^{3} L+U L U^{2}-2 U^{2} L^{3}+2 U L^{3} U+8 U L^{5}=\left[-2 U L^{2} U L-2(L U)^{2} L+2 L^{2} U^{2} L-8 U L^{5}\right. \\
& \left.-8 L U L^{4}-4 L^{3} U L^{2}+8 L^{4} U L+40 L^{7}\right]+U L U^{2}-2 U^{2} L^{3}+2 U L^{3} U+8 U L^{5} \\
= & -2 U L^{2} U L+\left[2 L U L^{2} U+2 L(L U)^{2}+\left[4 L^{3} U L^{2}+2 L^{4} U L+10 L^{5} U+40 L^{7}\right]\right. \\
& \left.+8 L U L^{4}+4 L^{2} U L^{3}-4 L^{3} U L^{2}-8 L^{5} U+48 L^{7}\right]+2 L^{2} U^{2} L-8 U L^{5}-8 L U L^{4} \\
& -4 L^{3} U L^{2}+8 L^{4} U L+40 L^{7}+U L U^{2}+\left[\left[-2 L^{2} U^{2} L+4 L U L^{4}-4 L^{4} U L-16 L^{7}\right]\right. \\
& \left.-4 U L^{5}+4 L^{3} U L^{2}+16 L^{7}\right]+2 U L^{3} U+8 U L^{5}
\end{aligned}
$$

that is,

$$
\begin{aligned}
& U L U^{2}=2\left(U L^{2} U L-U L^{3} U-L U L^{2} U-L^{2} U L U+\right. \\
& \left.2 U L^{5}-2 L U L^{4}-2 L^{2} U L^{3}-3 L^{4} U L-L^{5} U-16 L^{7}\right) .
\end{aligned}
$$

Next, we can reduce the relation $0=\delta[y, x, x, x x] x^{4}$ using the above identities. This yields

$$
U L^{5}=-L U L^{4}+\frac{1}{2} L^{2} U L^{3}+\frac{3}{4} L^{4} U L+\frac{3}{4} L^{5} U+8 L^{7} .
$$

Now combining (2.8) and (2.9) we obtain $U L U^{2}=2 U L U L-2 U L^{3} U-2 L U L^{2} U-$ $2 L^{2} U L U-8 L U L^{4}-2 L^{2} U L^{3}-3 L^{4} U L+L^{5} U$. Thus, we have three identities of $x$-degree 7 and $y$-degree 1 which may be written as multiplication identities:

Table iii. Multiplication identities of degree 7.

\begin{tabular}{lrrrrrrrrrr}
\hline & $U L^{2} U L$ & $U L^{3} U$ & $L U L U$ & $L(L U)^{2}$ & $L U L^{4}$ & $L U L^{3}$ & $L^{3} U L$ & $L^{4} U L$ & $L^{5} U$ & $L^{7}$ \\
\hline$L^{3} U$ & 0 & 0 & 0 & 0 & 0 & 0 & -2 & -1 & -5 & -20 \\
$U L^{5}$ & 0 & 0 & 0 & 0 & -1 & $1 / 2$ & 0 & $3 / 4$ & $3 / 4$ & 8 \\
$U L U^{2}$ & 2 & -2 & -2 & -2 & -8 & -2 & 0 & -3 & 1 & 0 \\
\hline
\end{tabular}

In an analogous way, using successively the identities

$$
0=\delta\left[y, x, x, x\left(x\left(x^{2} x\right)\right)\right] x^{4}, \quad 0=\delta\left[y, x, x, x^{2} x\right] x^{4}, \quad 0=\delta\left[y, x, x^{2}, x\left(x^{2} x\right)\right] x^{4},
$$

multiplying the second identity of Table ii with the operator $U$ from the left and replacing the occurrences of $U U L$, and finally using $0=\delta\left[y, x, x^{3}, x^{2} x\right] x^{4}$, we obtain the following 5 multiplication identities: 
Table iv. Multiplication identities of degree 8 .

\begin{tabular}{lrrrrrrrrr}
\hline & $U L^{4} U$ & $L U L^{2} U L$ & $L U L^{3} U$ & $L^{2} U L U$ & $L^{2} U L^{4}$ & $L^{4} U L^{2}$ & $L^{5} U L$ & $L^{6} U$ & $L^{8}$ \\
\hline$L^{3} U L U$ & 0 & 0 & 0 & 0 & 0 & $-1 / 2$ & -2 & $-11 / 2$ & -20 \\
$U L U^{2}$ & -4 & -2 & -2 & 0 & 2 & $-5 / 2$ & 13 & $31 / 2$ & 32 \\
$(U L)^{2}$ & 0 & 1 & 0 & -1 & -12 & $-11 / 4$ & $-7 / 2$ & $25 / 4$ & 36 \\
$U L^{3} U L$ & -1 & -1 & -1 & 0 & -4 & $-11 / 2$ & -3 & $9 / 2$ & 0 \\
$L^{3} U L^{3}$ & 0 & 0 & 0 & 0 & 0 & $-3 / 4$ & $-3 / 2$ & $-3 / 4$ & -8 \\
\hline
\end{tabular}

Now, relations $0=\delta\left[y, x, x^{3}, x\left(x^{2} x^{2}\right)\right] x^{4}, 0=\delta\left[y, x, x^{2}, x\left(x\left(x^{2} x^{2}\right)\right)\right] x^{4}, 0=\delta\left[y, x^{2}, x^{2}\right.$, $\left.\left(x^{2} x^{2}\right)\right] x^{4}, 0=\delta\left[y, x, x^{2} x^{2}, x^{2} x^{2}\right] x^{4}, 0=\delta\left[y, x^{2}, x^{3}, x^{2} x^{2}\right] x^{4}$, and multiplying the relation determined by the last row of Table iii with the operator $U$ from the left and first replacing the occurrences of $U U L$, imply the following 6 multiplication identities:

Table v. Multiplication identities of degree 9.

\begin{tabular}{lrrrr}
\hline & $L U L^{4} U$ & $\left(L^{2} U\right)^{2} L$ & $L^{7} U$ & $L^{9}$ \\
\hline$L^{6} U L$ & 0 & 0 & -7 & -48 \\
$L(L U)^{2}$ & 0 & 0 & -217 & $-4510 / 3$ \\
$U L^{4} U L$ & 1 & 0 & $-587 / 2$ & $-6155 / 3$ \\
$L^{2} U L^{3} U$ & 0 & 0 & $29 / 3$ & $422 / 9$ \\
$U L^{2} U L U$ & 0 & 0 & $1318 / 3$ & $27988 / 9$ \\
$L^{5} U L^{2}$ & 0 & 0 & -23 & $-496 / 3$ \\
\hline
\end{tabular}

The author used a simples MAPLE language program to check these identities. We now present a solution of a Conjecture of (Correa, Hentzel, Labra, 2002) in the positive. We see that for every $a \in A$, the associative algebra $A_{a}$, generated by the multiplication operators $L_{a}$ and $L_{a^{2}}$, is nilpotent of index $\leq 10$.

Theorem 1. Let $A$ be an algebra over a field $F$ of characteristic $\neq 2,3$, satisfying $x^{4}=0$. Then every monomial in $P$ of $x$-degree $\geq 10$ and $y$-degree 1 is an identity in $A$. In particular, $L_{a}^{10}=0$ for all $a \in A$.

Proof. First we shall prove that every monomial of $x$-degree 10 and $y$-degree 1 is an identity in $A$. Multiplying the operators in the first line of Table v with $L$ from the left and from the right, and the operators in the first line of Table iv with $U$ from the left and from the right and next using reductions from Tables $\mathrm{i}-\mathrm{v}$ we see that we only need to prove that $L^{2} U L^{4} U=0, L^{8} U=0$ and $L^{10}=0$ are multiplication identities in $A$. Now, for any $x$ in $A$ we have

$$
\begin{array}{cc}
{[l l] L^{7} U L} & =L\left(L^{6} U L\right)=-7 L^{8} U-48 L^{10} \\
L^{6} U L^{2} & =\left(L^{6} U L\right) L=-7 L^{7} U L-48 L^{10}=49 L^{8} U+288 L^{10} \\
L^{6} U L^{2} & =L\left(L^{5} U L^{2}\right)=-23 L^{8} U-496 / 3 L^{10}
\end{array}
$$

Therefore

$$
27 L^{8} U+170 L^{10}=0
$$


Now,

$$
\begin{gathered}
{[l l] L^{5} U L^{3}=\left(L^{5} U L^{2}\right) L=-23 L^{7} U L-496 / 3 L^{10}=161 L^{8} U+2816 / 3 L^{10}} \\
L^{5} U L^{3}=L^{2}\left(L^{3} U L^{3}\right)=-3 / 4 L^{6} U L^{2}-3 / 2 L^{7} U L-3 / 4 L^{8} U-8 L^{10} \\
=-27 L^{8} U-152 L^{10}
\end{gathered}
$$

and hence

$$
141 L^{8} U+818 L^{10}=0
$$

Next

$$
\begin{gathered}
{[l l] L^{3} U L^{3} U} \\
L^{3} U L^{3} U=L\left(L^{2} U L^{3} U\right)=29 / 3 L^{8} U+422 / 9 L^{10} \\
=\left(L^{3} U L^{3}\right) U=-3 / 4 L^{4} U L^{2} U-3 / 2 L^{5} U L U-3 / 4 L^{6} U U-8 L^{8} U \\
=-3 / 4 L\left(L^{3} U L^{2} U\right)-3 / 2 L^{2}\left(L^{3} U L U\right)-3 / 4 L^{3}\left(L^{3} U^{2}\right)-8 L^{10} \\
=9 / 4 L^{6} U L^{2}+15 / 4 L^{7} U L+667 / 4 L^{8} U+2345 / 2 L^{10} \\
=1003 L^{8} U+3281 / 2 L^{10}
\end{gathered}
$$

so that

$$
17880 L^{8} U+28685 L^{10}=0
$$

Combining (2.10-2.12) we obtain that $L^{8} U=0$ and $L^{10}=0$. Now, we have by Table $\mathrm{v}$ that $0=\left(L^{2} U L^{3} U\right) L=L^{2}\left(U L^{3} U L\right)=-L^{2} U L^{4} U-L^{3} U L^{2} U L-L^{3} U L^{3} U-4 L^{4} U L^{4}-$ $11 / 2 L^{6} U L^{2}-3 L^{7} U L+9 / 2 L^{8} U=-L^{2} U L^{4} U-\left(L^{3} U L^{2} U\right) L-4 L\left(L^{3} U L^{3}\right) L=-L^{2} U L^{4} U$. Therefore, we have $L^{2} U L^{4} U=0$.

In an analogous way, we can see that every monomial of $x$-degree 11 and $y$-degree 1 is an identity in $A$. This proves the theorem.

Now we shall investigate two subvarieties of $V$. We start in Subsection 2.1 with the class of all nilalgebras in $V$ of index $\leq 5$ and next in Subsection 2.2 we study the multiplication identities of the variety of all the nilalgebras in $V$ of index $\leq 6$.

\subsection{The identity $\mathrm{x}((\mathrm{xx})(\mathrm{xx}))=0$}

We will now consider the class of all algebras in $V$ satisfying the identity $x(x x)=0$. First, linearization $\delta[y]\left\{x\left(x^{2}\right)^{2}\right\}$ implies

$$
L_{x^{2} x^{2}}=-4 L U L
$$

and identity $\delta[y]\left\{x^{2} x^{3}\right\}=0$ forces

$$
U U=-2 U L L+2 L U L+4 L^{4} .
$$

Next, using above identity and $\delta\left[y, x^{2}\right]\left\{x\left(x^{2}\right)^{2}\right\}=0$ we get that $0=4 U U L+4 L U U+$ $8 L L_{x^{3}} L=4\left(U U L+L U U-2 L L U L-4 L^{5}\right)=8\left(-U L^{3}+L U L L+2 L^{5}-L U L L+L L U L+\right.$ 
$\left.2 L^{5}-L L U L-2 L^{5}\right)=8\left(-U L^{3}+2 L^{5}\right)$. Hence $U L^{3}=2 L^{5}$. Now idnetity $L_{x\left(x^{2} x^{2}\right)}=0$ and relations (2.5) and (2.14) imply $L^{2} U L=-L^{3} U-4 L^{5}$. Thus, we have the following multiplication identities.

Table vi. Multiplication identities of degree 5.

\begin{tabular}{lrrrr}
\hline & $U L U$ & $L U L^{2}$ & $L^{3} U$ & $L^{5}$ \\
\hline$U U L$ & 0 & 2 & 0 & 0 \\
$L U U$ & 0 & -2 & -2 & -4 \\
$L^{2} U L$ & 0 & 0 & -1 & -4 \\
$U L^{3}$ & 0 & 0 & 0 & 2 \\
\hline
\end{tabular}

From Table ii, we can prove that

$$
(U L)^{2}=-U L^{2} U-(L U)^{2}+2 L^{3} U L+4 L^{4} U+16 L^{6},
$$

and $\delta\left[x^{2}\right]\left\{x^{2}(x(x(x y)))-2 x(x(x(x(x y))))\right\}=0$ forces

$$
(U L)^{2}+U L^{2} U+2 L^{3} U L+4 L^{6}=0 .
$$

Combining (2.15) and (2.16), we have $(L U)^{2}=4 L^{6}$ and $(U L)^{2}=-U L^{2} U+2 L^{4} U+4 L^{6}$. Now, we can check easily the following multiplication identities.

\begin{tabular}{|c|c|c|c|c|c|c|c|}
\hline & $U L L U$ & $L^{4} U$ & $L^{6}$ & & $U L L U$ & $L^{4} U$ & $L^{6}$ \\
\hline$U U U$ & -2 & 4 & 8 & $L L U U$ & 0 & -4 & -4 \\
\hline$U U L L$ & 0 & 0 & 4 & $U L^{4}$ & 0 & 0 & 2 \\
\hline$U L U L$ & -1 & 2 & 4 & $L U L^{3}$ & 0 & 0 & 2 \\
\hline$L U U L$ & 0 & 2 & 0 & $L^{2} U L^{2}$ & 0 & 1 & 0 \\
\hline$L U L U$ & 0 & 0 & 4 & $L^{3} U L$ & 0 & -1 & -4 \\
\hline
\end{tabular}

Table vii. Multiplication identities of degree 6.

Theorem 2. Let $A$ be an algebra over a field $F$ of characteristic $\neq 2$ or 3 , satisfying the identities $x^{4}=0$ and $x\left(x^{2} x^{2}\right)=0$. Then every monomial in $P$ of $x$-degree $\geq 7$ and $y$-degree 1 is an identity in $A$. In particular, $L_{a}^{7}=0$ for all $a \in A$. Furthermore, the algebra generated by $L_{x}$ and $L_{x^{2}}$ is spanned, as vector space, by $L, U, L^{2}, U L, L U, L^{3}, U L^{2}, L U L, L^{2} U, L^{4}, U L U, L U L^{2}, L^{3} U, L^{5}, U L^{2} U, L^{4} U, L^{6}$.

Proof. We shall prove that every monomial of $x$-degree $\geq 7$ and $y$-degree 1 is an identity in $A$. Multiplying the operators in the first line of Table vii with $L$ and $U$ from the left and from the right, and the operators in the first line of Table vi with $U$ from the left and from the right, and next using reductions from Tables i-vii we see that we only need to prove that $L U L^{2} U=0, L^{5} U=0$ and $L^{7}=0$ are multiplication identities in $A$. Now, we have $0=\delta\left[y, x^{2} x^{2}\right]\left\{x\left(x^{2}\right)^{2}\right\}=4 L_{x^{2} x^{2}} U L+4 L U L_{x^{2} x^{2}}=-16 L U L U L-16 L U L U L=$ $-32 L U L U L=-32(L U)^{2} L=-2^{7} L^{7}$, so that $L^{7}=0$. Also $0=L U L U L=L(U L)^{2}=$ $-L U L^{2} U+2 L^{5} U$. Therefore, $L U L^{2} U=2 L^{5} U$. Finally, from Table vi we have that $0=\left(L^{2} U L+L^{3} U+4 L^{5}\right) L^{2}=L^{2} U L^{3}+L^{3} U L^{2}=L^{3} U L^{2}=L\left(L^{2} U L^{2}\right)=L^{5} U$. This proves the theorem. 


\subsection{The identity $\mathrm{x}(\mathrm{x}((\mathrm{xx})(\mathrm{xx})))=0$}

In this subsection we consider the class of all algebras in $V$ satisfying the identity $x(x(x x))=0$. Because we use linearization process of identities and $x\left(x\left(x^{2} x^{2}\right)\right)$ has degree 6 , we need consider the field $F$ of characteristic not 5 (2 or 3.)

From linearization $\delta[y]\{x(x(x x))\}$, we get the multiplication identity $L_{x\left(x^{2} x^{2}\right)}+L L_{x^{2} x^{2}}+$ $4 L^{2} U L=0$ and now Lemma 2 forces

$$
L U U=-2 L U L^{2}-2 L^{3} U-4 L^{5} .
$$

The relation $0=\delta\left[y, x^{2}\right]\left\{x\left(x\left(x^{2} x^{2}\right)\right)\right\}=U L_{x^{2} x^{2}}+4 L L^{x^{2} x^{3}} L+4 U L U L+4 L U U L+$ $8 L^{2} L_{x^{3}} L+4 L^{2} U U$ implies

$$
L U L^{3}=-\frac{1}{2}\left(L^{2} U L^{2}+L^{3} U L\right)
$$

since we can use identities from Tables i-v. Next, by $0=\delta\left[y, x^{3}\right]\left\{x\left(x\left(x^{2} x^{2}\right)\right)\right\}$ and $0=\delta\left[y, x^{2}, x^{2}\right]\left\{x\left(x\left(x^{2} x^{2}\right)\right)\right\}$ we get

$$
\begin{aligned}
{[l c l] L^{4} U L } & =-3 L^{5} U-16 L^{7} \\
L^{2} U L U & =-L^{3} U L^{2}+5 L^{5} U+28 L^{7},
\end{aligned}
$$

and identities $0=\delta\left[y, x^{2}, x, x\right]\left\{x\left(x\left(x^{2} x^{2}\right)\right)\right\}$ and $0=\delta\left[y, x^{2}, x^{3}\right]\left\{x\left(x\left(x^{2} x^{2}\right)\right)\right\}$ imply

$$
\begin{aligned}
{[l c l] U L^{4} U } & =-\frac{1}{2} L^{2} U L^{2} U+24 L^{6} U+62 L^{8}, \\
L^{2} U L^{2} U & =48 L^{6} U+156 L^{8} .
\end{aligned}
$$

Now, identity $0=\delta\left[y, x x^{2}\right]\left\{x x^{3}\right\}$ forces

$$
L^{6} U=-2 L^{8}
$$

Theorem 3. Let $A$ be a commutative algebra over a field $F$ of characteristic not 2,3 or 5 , satisfying the identities $x^{4}=0$ and $x\left(x\left(x^{2} x^{2}\right)\right)=0$. Then every monomial in $P$ of $x$-degree $\geq 9$ and $y$-degree 1 is an identity in $A$. In particular, $L_{a}^{9}=0$ for all $a \in A$.

Proof. By Tables i-v, we only need to prove that $L U L^{4} U=0, L^{2} U L^{2} U L=0$, $L^{7} U=0$ and $L^{9}=0$ are multiplication identities in $A$. From (2.19-2.23) may be deduced immediately $L^{7} U=-2 L^{9}$ and $2 L^{9}=2 L^{8} L=-L^{6} U L=-L^{2}\left(L^{4} U L\right)=$ $3 L^{7} U+16 L^{9}=-6 L^{9}+16 L^{9}=10 L^{9}$. Therefore $L^{9}=0$ and $L^{7} U=0$ are identities in A. Now $L^{2} U L^{2} U L=\left(L^{2} U L^{2} U\right) L=48 L^{6} U L+156 L^{9}=0$ and $L U L^{4} U=L\left(U L^{4} U\right)=$ $-(1 / 2) L^{3} U L^{2} U+24 L^{7} U+62 L^{9}=-(1 / 2) L\left(L^{2} U L^{2} U\right)=-24 L^{7} U-78 L^{9}=0$. This proves the theorem. 


\title{
References
}

[1] Albert, A. A.. Power-associative rings. Trans. Amer. Math. Soc. 64 : pp. 552-593, (1948).

[2] Correa, I., Hentzel I.R., Labra, A. (2002). On the nilpotence of the multiplication operator in commutative right nil algebras. Comm. in Algebra 30, pp. 3473-3488, (2002).

[3] Elduque, A., Labra, A.. On the Classification of commutative right-nilalgebras of dimension at most four. Comm. in Algebra 35 : pp. 577-588, (2007).

[4] Elgueta, L., Suazo, A.. Solvability of commutative power-associative nilalgebras of nilindex 4 and dimension $\leq 8$. Proyecciones 23 : pp. 123-129, (2004).

[5] Elgueta, L., Fernandez, J. C. G., Suazo, A.. Nilpotence of a class of commutative power-associative nilalgebras. Journal of Algebra 291 : pp. 492-504, (2005).

[6] Fernandez, J. C. G.. On commutative power-associative nilalgebras. Comm. in Algebra 32 : pp. 2243-2250, (2004).

[7] Fernandez, J. C. G., Suazo, A.. Commutative power-associative nilalgebras of nilindex 5. Result. Math. 47 : pp. 296-304, (2005).

[8] Gerstenhaber, M.. On nilalgebras and linear varieties of nilpotent matrices II. Duke Math. J. 27 : pp. 21-31, (1960).

[9] Osborn, J. M.. Varieties of algebras. Adv. in Math. 8 : pp. 163-396, (1972).

[10] Zhevlakov, K. A., Slinko, A. M., Shestakov, I. P., Shirshov, A. I.. Rings that are nearly associative. Academic Press, New York, (1982).

\author{
Juan C. Gutierrez Fernandez \\ Departamento de Matemtica-IME, \\ Universidade de So Paulo, \\ Caixa Postal 66281, \\ CEP 05315-970, \\ So Paulo, SP, \\ Brazil \\ e-mail : jcgf@ime.usp.br
}

